\title{
On Compatibility and Improvement of Different Quantum State Assignments
}

\author{
F Herbut $\dagger$ \\ Serbian Academy of Sciences and Arts, Knez Mihajlova 35, 11000 Belgrade, Serbia \\ and Montenegro
}

\begin{abstract}
When Alice and Bob have different quantum knowledges or state assignments (density operators) $\rho_{A}$ and $\rho_{B}$ respectively for one and the same specific individual system, then the problems of compatibility and pooling arise. The socalled first Brun-Finkelstein-Mermin (BFM) condition for compatibility is reobtained in terms of possessed or sharp (i. e., probability one) properties. The second BFM condition is shown to be generally invalid in infinite dimensional state space. An argument leading to a procedure of improvement of $\rho_{A}$ on account of $\rho_{B}$ and vice versa is presented.
\end{abstract}




\section{Introduction}

To my knowledge, the problem of compatible state assignments originated with Peierls 1]. His necessary conditions were seriously criticized be Fuchs and Mermin [2. Then Brun, Finkelstein and Mermin (BFM) 3], 4] derived two necessary conditions for compatibility of different state assignments $\rho_{A}$ and $\rho_{B}$, i. e., for two density operators, describing (being the quantum knowledge about) one and the same system. The two conditions were found to be equivalent in finite dimensional state space (to which they confined their discussion).

The first BFM condition reads that the intersection of the supports is at least one dimensional:

$$
\operatorname{dim}\left\{\operatorname{supp}\left(\rho_{A}\right) \cap \operatorname{supp}\left(\rho_{B}\right)\right\} \geq 1 \text {. }
$$

(Support of a density operator is the subspace spanned by the eigensubspaces corresponding to positive eigenvalues.)

The second BFM condition states that there exist pure-state expansions

$$
\begin{aligned}
& \rho_{A}=p_{A}|\phi\rangle\left\langle\phi\left|+\sum_{i \geq 1} p_{A i}\right| \phi_{A i}\right\rangle\left\langle\phi_{A i}\right|, \\
& \rho_{B}=p_{B}|\phi\rangle\left\langle\phi\left|+\sum_{i \geq 1} p_{B i}\right| \phi_{B i}\right\rangle\left\langle\phi_{B i}\right|
\end{aligned}
$$

(with all weights non-negative and $p_{A}$ and $p_{B}$ both positive) having a common pure state $|\phi\rangle$.

In reference [5] two approaches to compatibility are discussed. One is shown to be equivalent to the BFM condition. The other leads to a hierarchy of measurement-based compatibility criteria, all inequivalent with the BFM condition.

In reference [6] a more general approach based on a measure of the compatibility between two state assignments is expounded. This measure is then applied to a procedure of pooling information.

In reference [7] classical and quantum pooling of informations is discussed. The author claims that in the quantum case Alice and Bob must also possess information about how their respective states of knowledge were obtained.

Most likely there are some more or less important contributions that I have unintentionally omitted in this very short review because I am not aware of them.

In 44 a thorough list of further questions is given. Clearly, the problem of

compatibility and of pooling of information from different state assignments (for one and the same individual system) is not quite near to its complete solution.

Henceforth the state space is allowed to be finite or countably infinite dimensional.

This article is organized as follows: In section 2 the first BFM necessary condition is reobtained in a mathematically slightly but physically considerably different way than in the BFM article [3]. (The approach of section 2 is required for section 4.) In section 3 it is shown that in infinite dimensional state space the two BFM conditions are not equivalent, and that only the first one is generally valid. In section 4 a possible 
improvement of $\rho_{A}$ on account of $\rho_{B}$ and vice versa is expounded, and a simple way of pooling information from the two state assignments is given. Finally, the results of the article are summed up in a conclusion.

Needless to say that extension of the arguments of this article from two to any finite number of state assignments is straightforward.

\section{Derivation of the First BFM Condition}

When one is dealing with statistical knowledge, as it is the case with density operators, then one has in mind a random element of the ensemble. As far as a specific individual system from the ensemble is concerned, the statistical notions like the average hardly make sense.

If $P$ is a projector (physically: property or event), then $\operatorname{Tr} P \rho$ is the probability of possession of the property by (or of occurrence of the event on) a random system from the ensemble described by $\rho$. In the special case when $\operatorname{Tr}(P \rho)=1$, i. e., when one has a sharp or possessed property (a certain event), it is easy to show that for any state decomposition $\rho=\sum_{k} w_{k} \rho_{k}\left(\forall k: w_{k}>0, \rho_{k}>0, \operatorname{Tr} \rho_{k}=1 ; \sum_{k} w_{k}=1\right)$, all substates $\rho_{k}$ "inherit" the sharp property: $\forall k: \operatorname{Tr}\left(P \rho_{k}\right)=1$. Analogous statements hold true for finite laboratory ensembles and subensembles that represent empirically the density operators. Hence, in terms of sharp properties, one can speak of individualsystem knowledge, because it applies not only to a random, but also to a specific system in the ensemble.

If $P$ and $P^{\prime}$ are two projectors (commuting or not), one must clarify in what case they can both be simultaneously sharp properties of one and the same system.

It is shown in the Appendix that $P$ is a sharp property in the state $\rho$ if and only if:

$$
P \geq Q \text { meaning } P Q=Q \text {, }
$$

where $Q$ projects onto the support of $\rho$. (The first relation expresses implication in the lattice of projectors and the second is its algebraic equivalent).

Condition (3) makes it obvious that two properties $P$ and $P^{\prime}$ can both be simultaneously sharp properties of one and the same system if and only if their greatest lower bound $P_{\text {glb }}$ is nonzero, because then and only then do they have a common nonzero lower bound $Q$, which can be the support projector of a density operator. If $\left[P, P^{\prime}\right]=0$, then $P_{g l b}=P P^{\prime}$.

The following claim gives physical meaning to the greatest lower bound $P_{g l b}$.

Lemma 1. Properties $P$ and $P^{\prime}$ are sharp properties in a state $\rho$ if and only if so is their greatest lower bound $P_{g l b}$.

Proof follows immediately from the necessary and sufficient condition (3), because the two projectors have $Q$, the range projector of $\rho$, as their common lower bound if and only if $P_{g l b} \geq Q$.

Thus, $P$ and $P^{\prime}$ as sharp properties can be replaced by the single sharp property $P_{g l b}$. 
Returning to two state assignments $\rho_{A}$ and $\rho_{B}$ concerning one and the same system, the corresponding support projectors $Q_{A}$ and $Q_{B}$ are both sharp properties of the system at issue as seen from (3). Then so is their greatest lower bound $Q_{g l b}$, and it must not be zero, because zero cannot be a sharp property (cf (3)). $Q_{\text {glb }}$ projects onto $\operatorname{supp}\left(\rho_{A}\right) \cap \operatorname{supp}\left(\rho_{B}\right)$. Therefore, this subspace must not be zero either. This is the first BFM condition. It is obviously valid both in finite and in infinite dimensional state spaces.

\section{The Second BFM Condition in an Infinite Dimensional State Space}

An important result of Hadjisavvas 8] establishes the following claim.

Lemma 2. A pure state $|\phi\rangle$ can appear in a state decomposition of a given density operator $\rho(c f(2))$ in a state space of finite or infinite dimension if and only if

$$
|\phi\rangle \in \operatorname{ran}\left(\rho^{1 / 2}\right)
$$

where $\operatorname{ran}(. .$.$) denotes the range.$

If $\operatorname{supp}(\rho)$ is finite dimensional, then $\operatorname{ran}\left(\rho^{1 / 2}\right)=\operatorname{ran}(\rho)=\operatorname{supp}(\rho)$. But if $\operatorname{supp}(\rho)$ is infinite dimensional, then

$$
\operatorname{ran}(\rho) \subset \operatorname{ran}\left(\rho^{1 / 2}\right) \subset \operatorname{supp}(\rho)
$$

(proper subsets).

Let us take a simple example in which $\rho_{A}$ has an infinite-dimensional range and

$$
|\phi\rangle \in\left(\operatorname{supp}\left(\rho_{A}\right) \ominus \operatorname{ran}\left(\rho_{A}^{1 / 2}\right)\right), \quad\langle\phi|| \phi\rangle=1 .
$$

Let, further,

$$
|\phi\rangle \in \operatorname{supp}\left(\rho_{B}\right) .
$$

Let, finally,

$$
\left(\operatorname{supp}\left(\rho_{B}\right) \ominus \operatorname{span}(|\phi\rangle)\right) \perp\left(\operatorname{supp}\left(\rho_{A}\right) \ominus \operatorname{span}(|\phi\rangle)\right) .
$$

Then the first BFM condition is satisfied, but $|\phi\rangle$, the only common state vector (up to a phase factor) in the supports, cannot appear in a decomposition like the first one in (2) on account of Lemma 2. Therefore, the two BFM conditions are not equivalent if the support of at least one of the state assignments has an infinite dimensional support.

In view of Lemma 2, the second BFM condition is equivalent to

$$
\operatorname{dim}\left\{\operatorname{ran}\left(\rho_{A}^{1 / 2}\right) \cap \operatorname{ran}\left(\rho_{B}^{1 / 2}\right)\right\} \geq 1 .
$$

irrespectively of the dimensions of the supports. If both ranges are finite dimensional, (7) equals (1). If at least one of the ranges is infinite dimensional, the linear manifolds in (7) are proper subsets of the topologically closed subspaces appearing in (1). Then, (7) is stronger than (1), i. e., the former implies the latter, and I am not aware of any argument so far that would prove the validity of (7) as a necessary condition for compatibility of the two state assignments. 


\section{How Two Compatible State Assignments Can Improve Each Other}

We assume that (1) is valid, i. e., that the two state assignments are compatible. It may happen that $Q_{g l b} \equiv g l b\left(Q_{A}, Q_{B}\right)<Q_{A}$, i. e., that $Q_{g l b}$ is not a sharp property of the system at issue according to $\rho_{A}$, though it is known to be if also the information from $\rho_{B}$ is taken into account. In this case $\rho_{A}$ contains desinformation as far as the individual system under consideration is concerned, and one may like to dispense with it. We lean on the following mathematical facts in finding a way to do so.

Lemma 3. Let $P$ and $\rho$ be a projector and a density operator such that $p \equiv$ $\operatorname{Tr}(\rho P)>0$. Then $\rho_{L} \equiv P \rho P / p$ is closest to $\rho$ in the sense of Hilbert-Schmidt distance in comparison with all density operators for which $P$ is a sharp property.

The capital $L$ in the index is due to my liking to call $\rho_{L}$ a Lüders state. The reader is familiar with it in the context of change of state in ideal measurement. To my knowledge, it was introduced by Lüders [9] (and not by von Neumann [10] as many seem to think, cf also [1]). Its above claimed meaning concerning distance was established (in case of so-called non-selective measurement, when all the results of the measurement are taken into account) in previous work 12 .

Proof of Lemma 3 follows immediately if one takes into account the following facts: (i) All density operators are Hilbert-Schmidt ones, i. e., they are elements of the Hilbert space $\mathcal{H}_{H S}$ of Hilbert-Schmidt operators. These are linear operators $A$ such that $\operatorname{Tr}\left(A^{\dagger} A\right)<\infty$. (If the state space is finite dimensional, then all linear operators are Hilbert-Schmidt ones.) (ii) The superoperator $P \ldots P$ is a projector in $\mathcal{H}_{H S}$. (iii) The projection of a given vector into a given subspace of a (complex or real) unitary space has the smallest distance from the given vector in comparison with all vectors from the subspace.

The established claim of being "closest" may carry a mathematical elegance, but its physical meaning may be not so transparent. Therefore, we approach the Lüders state from another angle.

If $\rho_{A}$ is to be changed into another density operator describing a state in which a given property $P$ will be possessed, the statistical predictions will change in general. Still, there can be a set o predictions that should not change: those properties $P^{\prime}$ that imply $P$, so that when they become possessed, $P$ remains possessed.

Lemma 4. Let again $P, \rho$ be given with $p \equiv \operatorname{Tr}(\rho P)>0$. Let, further, $\mathcal{S} \equiv\left\{P^{\prime}: P \geq P^{\prime}\right\}$ be the set of all projectors implying $P$. Then

$$
\operatorname{Tr}\left(\rho P^{\prime}\right)=p\left[\operatorname{Tr}\left(\rho^{\prime} P^{\prime}\right)\right]
$$

for all $P^{\prime} \in \mathcal{S}$ if and only if $\rho^{\prime}=\rho_{L}$, where $\rho_{L}$ is the Lüders state (cf Lemma 3).

To my knowledge, a lemma related to the claim of Lemma 4 was first proved by Bell and Nauenberg in [13. We'll resort to their argument in the proof that follows.

Proof of Lemma 4. Let $\mathcal{S}^{\prime}$ be the subset of $\mathcal{S}$ containing all its projectors onto one-dimensional subspaces. They can be written as $|\psi\rangle\langle\psi|$. Then one can argue as 
follows

$$
\forall|\psi\rangle\langle\psi| \in \mathcal{S}^{\prime}: \quad \operatorname{Tr}[\rho(|\psi\rangle\langle\psi|)]=p\left\{\operatorname{Tr}\left[\rho^{\prime}(|\psi\rangle\langle\psi|)\right]\right\} \quad \Leftrightarrow\langle\psi|\rho| \psi\rangle=p\left\langle\psi\left|\rho^{\prime}\right| \psi\right\rangle .
$$

On the other hand,

$$
P|\psi\rangle=P\langle\psi \| \psi\rangle|\psi\rangle=\langle\psi \| \psi\rangle|\psi\rangle=|\psi\rangle
$$

Equivalently,

$$
|\psi\rangle \in \operatorname{supp}(P) \text {. }
$$

Obviously, (9) is not only necessary, but also sufficient for $|\psi\rangle\langle\psi| \in \mathcal{S}^{\prime}$.

Hence, the above chain of equivalences can be continued as follows.

$$
\Leftrightarrow\langle\psi|(P \rho P)| \psi\rangle=p\left\langle\psi\left|\rho^{\prime}\right| \psi\right\rangle
$$

Since $P \rho P$ is zero in the orthocomplement of $\operatorname{supp}(P)$, and $|\psi\rangle$ is an arbitrary state vector in this subspace, we finally have

$$
\rho^{\prime}=P \rho P / p
$$

as claimed.

One might still object that Lemma 4 tells about statistical predictions (that should not change). We have a fixed individual system from the ensemble described by $\rho_{A}$ in mind. Statistics may not be quite applicable. Let us return to the sharp properties. They do have individual-system meaning.

Lemma 5. Let $\overline{\mathcal{S}}$ be the set of all sharp properties $P^{\prime}$ in a given state $\rho$ and let $P$ be a statistically possible but not necessarily sharp property of the random system in the state $\rho$, i. e., let $p \equiv \operatorname{Tr}(\rho P)>0$. Let, finally, $\overline{\mathcal{S}}^{\prime}$ be the subset of $\overline{\mathcal{S}}$ containing all $P^{\prime}$ compatible with $P$ as obseravables, $i$. e., for which $\left[P^{\prime}, P\right]=0$. Then both $P$ and each $P^{\prime}$ from $\overline{\mathcal{S}}^{\prime}$ are sharp properties in a state $\rho^{\prime}$ if and only if $\rho^{\prime}=P \rho P / p$.

Proof. The claim of Lemma 5 is a special case of a wider claim proved in [11] as Theorem 1 there. (The context was ideal measurement. But this was not relevant for the somewhat intricate proof given there.)

Returning to the two state assignments $\rho_{A}$ and $\rho_{B}$ and to the desinformation in the former, in view of Lemmata 3, 4, and 5, the desinformation can be dispensed with or $\rho_{A}$ can be improved if it is replaced by

$$
\bar{\rho}_{A} \equiv Q_{g l b} \rho_{A} Q_{g l b} / p_{g l b}^{A}
$$

where $Q_{g l b}$ is the greatest lower bound (in the lattice of projectors) of $Q_{A}$ and $Q_{B}$, the support projectors of $\rho_{A}$ and $\rho_{B}$ respectively, and $p_{g l b}^{A} \equiv \operatorname{Tr}\left(\rho_{A} Q_{g l b}\right)$. This probability is necessarily positive as proved in what follows.

Lemma 6. If $\rho$ is a density operator with $Q$ as its support projector, and $P$ is another nonzero projector implying $Q$, then the probability $\operatorname{Tr}(\rho P)$ is positive. 
Proof. Let us start ab contrario assuming that $\operatorname{Tr}(\rho P)=0$. We show that $P$ is then necessarily a subsprojector of or, equivalently, that it implies the null projector $(1-Q)$ of $\rho$, in contradiction to the assumptions in Lemma 6. To prove this, we write down a spectral form $\rho=\sum_{i} r_{i}|i\rangle\langle i|$ of $\rho$ in terms of its positive eigenvalues and the corresponding eigenvectors, and analogously for $P: P=\sum_{k}|k\rangle\langle k|$. Then

$$
0=\operatorname{Tr}(\rho P)=\sum_{i} \sum_{k} r_{i}|\langle i \| k\rangle|^{2} .
$$

Since all terms are non-negative, all $|k\rangle$ must be orthogonal to all $|i\rangle$.

Let us take stock of what has been achieved.

\section{How Much Improvement Has Been Achieved?}

Naturally, the symmetric expression to $(10)$, i. e., $\bar{\rho}_{B}=Q_{g l b} \rho_{B} Q_{g l b} / p_{\text {glb }}^{B}$ with $p_{g l b}^{B} \equiv$ $\operatorname{Tr}\left(\rho_{B} Q_{g l b}\right)$, is the improvement of $\rho_{B}$. It is desirable to clarify if there are distinct sharp properties in $\bar{\rho}_{A}$ and $\bar{\rho}_{B}$. Utilizing criterion (3), we take resort to the corresponding support projectors $\bar{Q}_{A}$ and $\bar{Q}_{B}$.

Theorem 1. The improved states $\bar{\rho}_{A}$ and $\bar{\rho}_{B}$ have one and the same support projector, i. e., $\bar{Q}_{A}=\bar{Q}_{B}=Q_{g l b}$.

Proof. It is obvious from (10) that $Q_{g l b} \bar{\rho}_{A}=\bar{\rho}_{A}$. Taking the trace, we see that $Q_{g l b}$ is a sharp property in the state $\bar{\rho}_{A}$. From (3) mutatis mutandis it follows that $Q_{g l b}-\bar{Q}_{A}$ is a projector. Evaluating $\operatorname{Tr}\left[\left(Q_{g l b}-\bar{Q}_{A}\right) \bar{\rho}_{A}\right]$, we obtain zero. On the other hand, due to $\left(Q_{g l b}-\bar{Q}_{A}\right) \leq Q_{g l b}$, or equivalently $\left(Q_{g l b}-\bar{Q}_{A}\right) Q_{g l b}=\left(Q_{g l b}-\bar{Q}_{A}\right)$, due to $(10)$, and commutation under the trace, we, further, obtain

$$
0=\operatorname{Tr}\left[\left(Q_{g l b}-\bar{Q}_{A}\right) \bar{\rho}_{A}\right]=\operatorname{Tr}\left[\left(Q_{g l b}-\bar{Q}_{A}\right) \rho_{A}\right] .
$$

Taking into account that $\left(Q_{g l b}-\bar{Q}_{A}\right) \leq Q_{g l b}$ and the latter is a subprojector of the support projector of $\rho_{A}$, hence also $\left(Q_{g l b}-\bar{Q}_{A}\right)$ is a subprojector of the same, we see that relation (11) and Lemma 6 imply $Q_{g l b}-\bar{Q}_{A}=0$.

Thus, the two improved states have the same set of sharp properties. The method of sharp properties applied so far cannot lead us any further. This all generalizes trivially to the case of several state assignments.

If one of the improved state assignments turns out to be pure, then all are, and all are the same pure state. With less luck in the pooling performed so far, one could end up with mixed improved states, but still all equal ones. This would also end the necessity for further pooling. But in general the improved states can be mixed and distinct. Then further pooling is required.

A simple way of pooling information from two state assignments goes as follows. Let $0<w<1$. The result of pooling is obtained by averaging

$$
\rho \equiv w Q_{g l b} \rho_{A} Q_{g l b} / p_{g l b}^{A}+(1-w) Q_{g l b} \rho_{B} Q_{g l b} / p_{g l b}^{B} .
$$

If the "quantum knowledges" of both Alice and Bob are believed equally trustworthy, then $w=1 / 2$ seems in order. 
A more sophisticated way of pooling is required in some cases (see e. g. [6] [7]). CONCLUSION. The basic method of this article is that of sharp properties. These are interpreted as being possessed by the individual quantum system about which Alice and Bob have quantum knowledges or state assignments. Making use of this method, the first BFM necessary condition (see the Introduction) was rederived. It was shown that in case of infinite-dimensional ranges of the density operators, the first BFM condition is valid; the second need not be. Finally, the method was utilized to improve Alice's and Bob's state assignments on account of information from each other. Thus, they end up with possibly different density operators, but they have one and the same set of sharp or possessed properties.

\section{Appendix}

We prove now that for a projector $P$ and a statistical operator $\rho$ with the range projector $Q$ one has $P$ as a sharp property, i. e., $\operatorname{Tr} P \rho=1$, if and only if $P \geq Q$.

Sufficiency. The assumed relation $P \geq Q$ means $P Q=Q$. Further, $Q \rho=\rho$. Hence,

$$
1=\operatorname{Tr} \rho=\operatorname{Tr}(Q \rho)=\operatorname{Tr}(P Q \rho)=\operatorname{Tr}(P \rho) .
$$

Necessity. Let

$$
\rho=\sum_{i} r_{i}|i\rangle\langle i|, \quad \forall i: \quad r_{i}>0,
$$

be a spectral form of $\rho$. By assumption, now one has

$$
1=\sum_{i} r_{i} \operatorname{Tr}(P|i\rangle\langle i|)=\sum_{i} r_{i}\langle i|P| i\rangle .
$$

Subtracting this from $1=\sum_{i} r_{i}$, one obtains

$$
0=\sum_{i} r_{i}(1-\langle i|P| i\rangle) .
$$

Since always $0 \leq\langle i|P| i\rangle \leq 1$, we have

$$
\forall i: \quad\langle i|P| i\rangle=1
$$

or equivalently,

$$
\begin{aligned}
\forall i:\left\langle i\left|P^{\perp}\right| i\right\rangle=0 & \Leftrightarrow \| P^{\perp}|i\rangle \|^{2}=0 \Leftrightarrow P^{\perp}|i\rangle=0 \\
& \Leftrightarrow P|i\rangle=|i\rangle .
\end{aligned}
$$

Since, $\sum_{i}|i\rangle\langle i|=Q$, we, finally, have $P Q=Q$ as claimed.

[1] R.E. Peierls, in: Symposium on the Foundations of Modern Physics, editors: P. Lahti and P. Mittelstaedt (World Scientific, Singapore, 1985) p.195;

R.E. Peierls, Phys. World, January, 19 (1991).

R. E. Peierls, More Surprises in Theoretical Physics (Princeton University Press, Princeton, New Jersey, 1991) p. 84. 
[2] N. D. Mermin, in: Quantum (Un)speakables: Essays in Commemoration of John S. Bell, editors: R. Bertlmann and A. Zeilinger (Springer Verlag, Berlin, 2001); also available as quant-ph/0107151.

[3] T. A. Brun, J. Finkelstein, N. D. Mermin, Phys Rev. A 65, 032315 (2002); also available as quant-ph/0109041.

[4] T. A. Brun, "How Much State Assignments Can Differ", quant-ph/0208088

[5] C. M. Caves, Ch. A. Fuchs, and R. Schack, Conditions for Compatibility of Quantum State Assignments, quant-ph/0206110

[6] D. Poulin and R. Blume-Kohout, Compatibility of Quantum States, quant-ph/0205033

[7] K. Jacobs, How Do Two Observers Pool Their Knowledge About a Quantum System?, quant-ph/0201096.

[8] N. Hadjisavvas, Lett. Math. Phys. 5, 327 (1981).

[9] G. Lüders, Ann. der Physik (Leipzig) 8, 322 (1951).

[10] J. von Neumann, Mathematical Foundations of Quantum Mechanics (Princeton University Press, Princeton, 1955).

[11] F. Herbut, Int. J. Theor. Phys. 11, 193 (1974).

[12] F. Herbut, Ann. Phys. 55271 (1969).

[13] J.S. Bell and M. Nauenberg, in: Preludes in Theoretical Physics, editors: A. De-Shalit, H. Feshbach, and L. van Hove (North-Holland Publ. Comp., Amsterdam, 1966), p.279. 